\title{
Highly sensitive SPR response of Au/chitosan/graphene oxide nanostructured thin films toward $\mathrm{Pb}$ (II) ions
}

\begin{abstract}
Optical sensors based on surface plasmon resonance (SPR) are utilized for detecting toxic heavy metals in solutions. To improve the sensitivity of SPR sensors, nanostructured thin films with active layers can be synthesized. In this study, the response to $\mathrm{Pb}$ (II) was measured and compared for SPR sensors incorporating gold-chitosan-graphene oxide $(\mathrm{Au} / \mathrm{CS} / \mathrm{GO})$ nanostructured thin films and $\mathrm{Au} / \mathrm{CS}$ films. The characterization of $\mathrm{Au} / \mathrm{CS} / \mathrm{GO}$ using FESEM analysis revealed a film composed of nanosheets with wrinkled, rough surfaces. The results from XRD analysis confirmed the successful incorporation of GO in the prepared films. Additionally, AFM analysis determined that the Au/CS/GO films had a root mean square (rms) roughness of $28.38 \mathrm{~nm}$ and were considerably rougher than the Au/CS films. Upon exposure to a $5 \mathrm{ppm} \mathrm{Pb}$ (II) ion solution, the $\mathrm{Au} / \mathrm{CS} / \mathrm{GO}$ films exhibited higher SPR sensitivity, as much as $1.11200 \mathrm{ppm}-1$, than $\mathrm{Au} / \mathrm{CS}$ films, $0.77600 \mathrm{ppm}-1$. This enhancement of the SPR response was attributed to strong covalent bonding between CS and $\mathrm{GO}$ in these films. These results indicated that the Au/CS/GO films show potential for the detection of heavy metal pollution in environmental applications.
\end{abstract}

Keyword: Surface plasmon resonance; Nanostructured thin film; Chitosan; Graphene oxide; Lead ion 